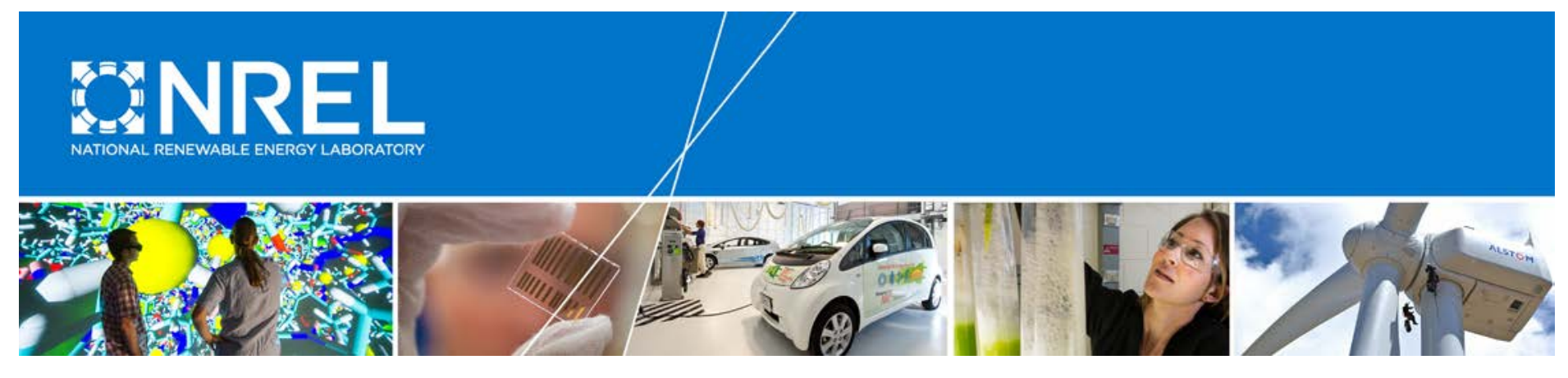

\title{
Solar Power Ramp Events Detection Using an Optimized Swinging Door Algorithm
}

\section{Preprint}

Mingjian Cui ${ }^{1}$, Jie Zhang ${ }^{2}$, Anthony Florita ${ }^{2}$, Bri-Mathias Hodge ${ }^{2}$, Deping $\mathrm{Ke}^{1}$, and Yuanzhang Sun ${ }^{1}$

${ }^{1}$ Wuhan University

${ }^{2}$ National Renewable Energy Laboratory

Presented at the ASME International Design Engineering Technical Conferences \& Computers and Information in Engineering Conference Boston, Massachusetts August 2-5, 2015

NREL is a national laboratory of the U.S. Department of Energy Office of Energy Efficiency \& Renewable Energy Operated by the Alliance for Sustainable Energy, LLC

This report is available at no cost from the National Renewable Energy Laboratory (NREL) at www.nrel.gov/publications.

Conference Paper

NREL/CP-5D00-64093

August 2015

Contract No. DE-AC36-08GO28308 


\section{NOTICE}

The submitted manuscript has been offered by an employee of the Alliance for Sustainable Energy, LLC (Alliance), a contractor of the US Government under Contract No. DE-AC36-08GO28308. Accordingly, the US Government and Alliance retain a nonexclusive royalty-free license to publish or reproduce the published form of this contribution, or allow others to do so, for US Government purposes.

This report was prepared as an account of work sponsored by an agency of the United States government. Neither the United States government nor any agency thereof, nor any of their employees, makes any warranty, express or implied, or assumes any legal liability or responsibility for the accuracy, completeness, or usefulness of any information, apparatus, product, or process disclosed, or represents that its use would not infringe privately owned rights. Reference herein to any specific commercial product, process, or service by trade name, trademark, manufacturer, or otherwise does not necessarily constitute or imply its endorsement, recommendation, or favoring by the United States government or any agency thereof. The views and opinions of authors expressed herein do not necessarily state or reflect those of the United States government or any agency thereof.

This report is available at no cost from the National Renewable Energy Laboratory (NREL) at www.nrel.gov/publications.

Available electronically at SciTech Connect http:/www.osti.gov/scitech

Available for a processing fee to U.S. Department of Energy and its contractors, in paper, from:

U.S. Department of Energy

Office of Scientific and Technical Information

P.O. Box 62

Oak Ridge, TN 37831-0062

OSTI http://www.osti.gov

Phone: 865.576.8401

Fax: 865.576.5728

Email: reports@osti.gov

Available for sale to the public, in paper, from:

U.S. Department of Commerce

National Technical Information Service

5301 Shawnee Road

Alexandria, VA 22312

NTIS http://www.ntis.gov

Phone: 800.553 .6847 or 703.605 .6000

Fax: 703.605.6900

Email: orders@ntis.gov 


\section{DETC2015-46849}

\section{SOLAR POWER RAMP EVENTS DETECTION USING AN OPTIMIZED SWINGING DOOR ALGORITHM}

\author{
Mingjian Cui ${ }^{1}$ \\ Wuhan University \\ Wuhan, Hubei 430072, China \\ Email: mj_cui@whu.edu.cn \\ Bri-Mathias Hodge 4 \\ National Renewable Energy Laboratory \\ Golden, Colorado 80401, USA \\ Email: bri.mathias.hodge@nrel.gov
}

\author{
Jie Zhang ${ }^{2}$ \\ National Renewable Energy Laboratory \\ Golden, Colorado 80401, USA \\ Email: jie.zhang@nrel.gov \\ Deping $\mathrm{Ke}^{5}$ \\ Wuhan University \\ Wuhan, Hubei 430072, China \\ Email: kedeping@whu.edu.cn
}

\author{
Anthony Florita ${ }^{3}$ \\ National Renewable Energy Laboratory \\ Golden, Colorado 80401, USA \\ Email: anthony.florita@nrel.gov \\ Yuanzhang Sun ${ }^{6}$ \\ Wuhan University \\ Wuhan, Hubei 430072, China \\ Email: yzsun@mail.tsinghua.edu.cn
}

\section{ABSTRACT}

Solar power ramp events (SPREs) significantly influence the integration of solar power on non-clear days and threaten the reliable and economic operation of power systems. Accurately extracting solar power ramps becomes more important with increasing levels of solar power penetrations in power systems. In this paper, we develop an optimized swinging door algorithm (OpSDA) to enhance the state of the art in SPRE detection. First, the swinging door algorithm (SDA) is utilized to segregate measured solar power generation into consecutive segments in a piecewise linear fashion. Then we use a dynamic programming approach to combine adjacent segments into significant ramps when the decision thresholds are met. In addition, the expected SPREs occurring in clear-sky solar power conditions are removed. Measured solar power data from Tucson Electric Power is used to assess the performance of the proposed methodology. OpSDA is compared to two other ramp detection methods: the SDA and the L1-Ramp Detect with Sliding Window (L1-SW) method. The statistical results show the validity and effectiveness of the proposed method. OpSDA can significantly improve the performance of the SDA, and it can perform as well as or better than L1-SW with substantially less computation time.

\footnotetext{
${ }^{1}$ Doctoral Candidate, School of Electrical Engineering (SEE); and Visiting Scholar, Transmission and Grid Integration Group, National Renewable Energy Laboratory.

${ }^{2}$ Research Engineer, Transmission and Grid Integration Group, ASME Member, Corresponding author.

${ }^{3}$ Research Engineer, Transmission and Grid Integration Group, ASME Member.

${ }^{4}$ Senior Research Engineer, Transmission and Grid Integration Group.

${ }^{5}$ Lecturer, School of Electrical Engineering (SEE).

${ }^{6}$ Professor, School of Electrical Engineering (SEE).
}

Keywords: Dynamic programming, ramp forecasting, solar power ramp events, swinging door algorithm, Tucson Electric Power

\begin{tabular}{cl} 
& \multicolumn{1}{c}{ NOMENCLATURE } \\
$J$ & objective function to be maximized \\
$S(i, j)$ & score function of the time interval $(i, j)$ \\
$R(i, j)$ & rule sets of the time interval $(i, j)$ \\
$R_{C}(i, j)$ & rule sets of the time interval $(i, j)$ in clear-sky \\
$\Theta$ & time interval set of SPREs and non-SPREs \\
$\xi$ & time interval set of SPREs \\
$\bar{\xi}$ & time interval set of non-SPREs \\
$E_{(\cdot)}$ & time interval of one SPRE \\
\hline$E_{(\cdot)}$ & time interval of one non-SPRE \\
$p_{t}$ & solar power generation at the time $t$ \\
$S_{(\cdot)}$ & start time of one SPRE \\
$e_{(\cdot)}$ & end time of one SPRE \\
\hline$S_{(\cdot)}$ & start time of one non-SPRE \\
\hline$e_{(\cdot)}$ & end time of one non-SPRE \\
$m$ & index of the $m$ th SPRE \\
$L$ & number of solar power data points \\
$M$ & number of SPREs \\
$\varepsilon$ & only tunable parameter in SDA \\
$\lambda$ & penalty parameter in L1-SW \\
$\gamma$ & second derivative threshold in L1-SW
\end{tabular}

\section{INTRODUCTION}

The increasing penetration of solar power in the United States has presented new challenges for the reliable and economic operations of the electric grid because of the high variability and uncertainty of solar power [1-3]. Solar power ramp events 
(SPRE) are derived from large fluctuations in solar power, especially in a very short time [4]. Some of these ramp events [5] are caused by changes in short-term microclimates, such as passing clouds, which are not predictable as diurnal variability. When a large SPRE occurs, it can be challenging to maintain and manage a power system's balance. During severe solar ramp events, power system operators have to take measures to compensate for the significant changes in solar power. These measures include modulating the outputs of traditional generators (especially the thermal and hydroelectric units), using grid ancillary services, and even curtailing or restricting the output of solar generators without considering the economic consequences. Accurately predicting and identifying SPREs reduces the influence of solar power ramps and thereby increases the dispatchability of solar power.

Solar power ramp forecasting is still a relatively new research topic. However, a significant amount of work has been done in solar irradiance and power forecasting, which provides useful information for solar power ramp forecasting. Zhang et al. [6] analyzed the sensitivity of a suite of metrics to solar forecasts with uniform improvement, ramp forecasting improvements, and a ramp forecasting threshold. Bacher et al. [7] utilized statistical smoothing techniques and adaptive linear time series models to perform online forecasting of short-term solar power. Bessa et al. [8] presented a spatial-temporal model based on a vector autoregressive framework to forecast solar power. It took advantage of a smart grid infrastructure with smart meters and advanced control functions. Yang et al. [9] presented a weatherbased hybrid method for day-ahead hourly forecasting of solar power output. The method consisted of classification, training, and forecasting stages. Yang et al. [10] proposed a multitimescale data-driven forecast model that involved the spatial and temporal correlations among neighboring solar sites to improve the accuracy of solar power forecasting.

Several studies of SPRE forecasting can be found in the literature. Florita et al. [11] used the swinging door algorithm (SDA) to identify variable generation ramping events from historical solar power data. Hummon et al. [12] analyzed solar power ramping in the state of Gujarat in India using highresolution solar data and found that the total magnitude of solar power ramping goes up with increased solar capacity. Hodge et al. [13] analyzed solar ramp distributions at different timescales and weather patterns. Sengupta and Keller [14] analyzed SPREs at individual locations and provided a comprehensive analysis of solar variability for a distributed generation scenario.

In this research, a novel optimized swinging door algorithm (OpSDA) is developed to detect SPREs. The proposed OpSDA enhances the performance of the SDA through the introduction of dynamic programming in the ramp detection process. The SDA has been widely used in the literature for ramp forecasting. The SDA was originally proposed by Bristol [15] for data compression and has been recently used in the renewable energy community. Zhang et al. [16] adopted the SDA to extract ramp events from actual and forecasted wind power and evaluate the ramp forecasting performance of improved short-term wind power forecasts. Makarov and Ma et al. [17-20] used the SDA to derive three parameters for each power interval: ramping capability, ramping rate, and ramping duration. Cui et al. [21] proposed an OpSDA to improve wind power ramp detection performance.

Dynamic programming is a method for solving a complex problem by breaking it down into a collection of simpler subproblems. It is applicable to problems exhibiting the properties of overlapping subproblems and optimal substructures. Sevilian and Rajagoapal [22] defined a family of scoring functions with ramp event definitions and used a dynamic programming recursion technique to detect all the ramp events. Boulaxis and Papadopoulos [23] utilized a dynamic programming technique to solve the optimal feeder routing of distribution networks. Marano et al. [24] coupled a dynamic programming algorithm with a mathematical model to achieve the optimal management of a compressed air energy storage plant.

The proposed OpSDA for identifying solar power ramps is summarized as follows:

1) The SDA is utilized to segregate solar power data into piecewise segments, and then all these piecewise segments are merged and optimized through a dynamic programing process. To apply the dynamic programming process, the solar power signal is divided into a number of overlapping windows in which a ramp score function is defined to perform the recursion of the dynamic programming algorithm and remove ramps that also occur in clear-sky power generation.

2) Based on the OpSDA detection method, the only tunable parameter, $\varepsilon$, of the SDA can be ascertained as an optimal parameter for the online solar ramp detection. A suite of metrics are applied to evaluate the performance of ramp extraction with 10 different $\varepsilon$ values and determine the optimal value, $\varepsilon_{\text {opt }}$.

The following topics are discussed in the remainder of the paper: (i) the formulation of the OpSDA, which applies a dynamic programming approach to the SDA; (ii) a case study of Tucson Electric Power (TEP), which compares the OpSDA to the L1-Ramp Detect with Sliding Window (L1-SW) method; and (iii) a further application of the OpSDA as a benchmark to tune the optimal value, $\varepsilon_{\text {opt }}$, in the SDA.

\section{OPTIMIZED SWINGING DOOR ALGORITHM}

\section{Swinging Door Algorithm (SDA)}

The SDA $[15,25]$ has been recently used in the literature to extract ramp periods in a time series of a power signal. The SDA is based on the concept of a "swinging door" with a "turning point" (i.e., at time 0 with magnitude 5, as shown in Fig. 1) whenever the next point in the time series causes any intermediate point to fall outside the area partitioned by the up and down segment bounds. The segment bounds are defined by the door width, $\pm \varepsilon$, which is the only tunable parameter in the SDA. More detailed descriptions of the SDA can be found in [11, 16, 17]. For instance, points $\mathrm{A}, \mathrm{B}$, and $\mathrm{C}$ are all inside the segment bounds determined by Point $\mathrm{D}$ within $\pm \varepsilon$. After segregating the power signal by the SDA, SPREs are extracted according to the user-specified definition of a significant ramp. 


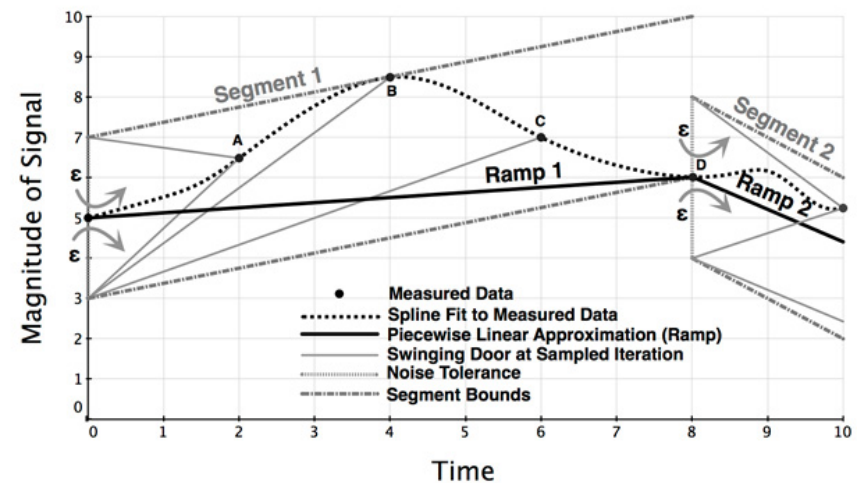

FIGURE 1. SDA FOR THE EXTRACTION OF RAMPS IN THE POWER SIGNAL [11]

Fig. 2 shows one example of ramp detection results by the SDA. As shown, there are two down-ramps from Point 38 to Point 41 (points 38 40 and points 40 41); however, there should be only one significant ramp event from Point 38 to Point 41 . Another case is from Point 44 to Point 47. Although there is actually one ramp event in the interval from points $44 \sim 47$, the start time detected by the SDA is Point 45 rather than Point 44, which is the measured start time of this ramp event. Similar circumstances can be seen in the intervals from points 34 37 and 41 43. Moreover, the significant ramp event in the interval from points 30 33 is not detected by the SDA, because each small segment within this interval does not comply with the ramp definition. However, if the three small segments within the interval from points 30 33 were combined together into one expanded segment, it could be a significant ramp event according to the ramp definition. Therefore, it is uniquely helpful to introduce optimization techniques into the SDA to achieve more accurate ramp detection. This motivates the development of the OpSDA, which is described in the next subsection.

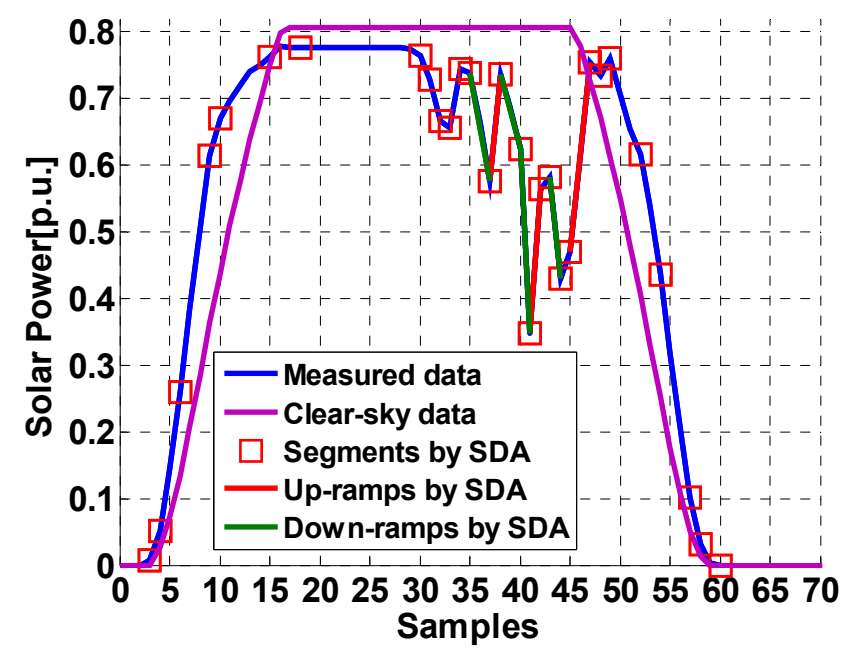

FIGURE 2. DETECTED SPRES BY THE SDA AND ITS CORRESPONDING SEGMENTS WITH $\varepsilon=0.9 \%$ AND 15MINUTE RESOLUTION

\section{OpSDA Based on a Dynamic Programming Approach}

The objective of the optimization is to minimize the number of individual ramps by combining adjacent small ramps.
Therefore, adjacent segments that have the same ramping direction (e.g., up-ramps or down-ramps) can be merged into one segment. Toward this end, a dynamic programming algorithm is applied to the original segments (from the SDA). In this study, an increasing length score function, $S$, was designed based on the length of the interval segregated by the SDA. The optimization seeks to maximize the length score function, which corresponds to a ramp event.

Given a solar power interval, $(i, j)$, of all discrete time points and an objective function, $J$, of the dynamic programming algorithm, a SPRE is detected by maximizing the objective function:

$$
J(i, j)=\max _{i<k \leq j}[S(i, k)+J(k, j)], i<j
$$

s.t.

$$
\begin{aligned}
& S(i, j)>S(i, k)+S(k+1, j), \forall i<k<j \\
& S(i, j)=(j-i)^{2} \times R(i, j) \times\left[1-R_{C}(i, j)\right]
\end{aligned}
$$

where $J(i, j)$ is the maximum score in the interval, $(i, j)$, which can be computed as the maximum over $(i-j)$ subproblems. $J(i, j)$ is constrained by the inequality constraint in Eq. (2) and the equality constraint in Eq. (3). Eq. (2) is a super-additivity property to which the positive score value, $S(i, k)$, must conform. Eq. (3) is improved based on [22] with the use of a new variable, $R_{C}(i, j)$. Except for the equality constraint with $R_{C}(i, j)$ in Eq. (3), OpSDA and L1-SW share the same variables and constraints. $R(i, j)$ and $R_{C}(i, j)$ are the definition of a ramp within the interval, $(i, j)$, in measured data days and clear-sky days, respectively. Similar definitions of $R(i, j)$ and $R_{C}(i, j)$ in Eq. (3) have been used in the literature $[26,27]$ for wind power ramp detection. Generally, significant changes of solar power magnitude, direction, and duration can result in a SPRE. In this paper, we use three classic definitions of significant ramp events that were originally defined for significant wind power ramp events [16]:

(i) Significant Ramp Definition 1: The change in solar power output that is greater than $10 \%$ of the installed solar capacity.

(ii) Significant Ramp Definition 2: The change in solar power output that is greater than $10 \%$ of the installed solar capacity within a time span of 1 hour or less.

(iii) Significant Ramp Definition 3: A significant up-ramp is defined as the change in solar power output that is greater than $10 \%$ of solar capacity within a time span of 1 hour or less; and a significant down-ramp is defined as the change in solar power output that is greater than $8 \%$ of solar power capacity within a time span of 1 hour or less.

First, if $R(i, j)$ and $R_{C}(i, j)$ separately conform to the threshold of ramp definitions in measured data days and clearsky days, $R(i, j)$ and $R_{C}(i, j)$ are assigned to be 1 ; otherwise, $R(i, j)$ and $R_{C}(i, j)$ are assigned to be 0 . Second, a comparison process is made based on Eq. (3) to remove the SPREs likewise occurring in the clear-sky days (i.e., SPREs caused by the solar diurnal variation), in which $S(i, j)$ should be assigned to be 0 . This comparison process is implemented based on the algorithm developed in [22], which is given in Algorithm 1 with pseudocode. The main contribution of this research in Algorithm 1 (compared to [22]) is the introduction of a modified score 
function, $S$, to remove insignificant ramps occurring both in the measured and clear-sky solar power generations. The OpSDA has the same initialization code as L1-SW in [22]. The main difference between them is how to compute maximum scores for combined segments in Algorithm 1. A flowchart is provided in Fig. 3 to illustrate the ramp detection process of the OpSDA.

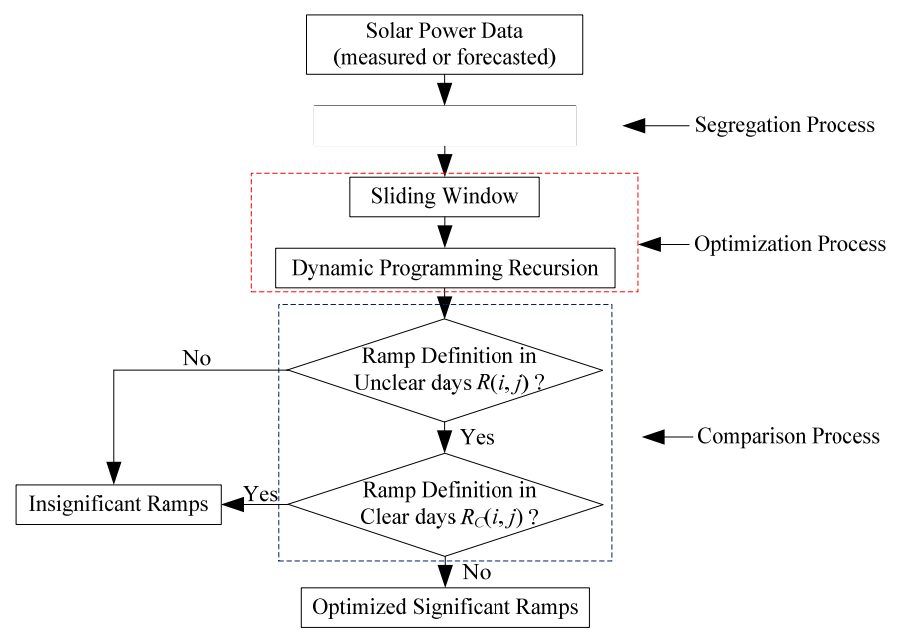

FIGURE 3. THE OVERALL PROCESS OF THE OPSDA

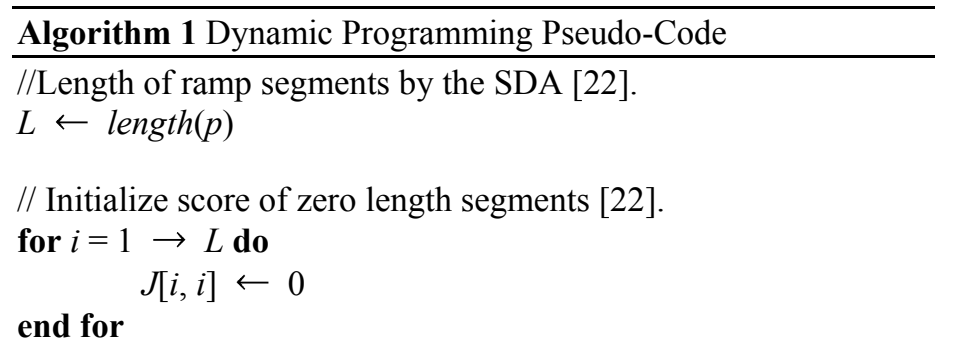

// Compute maximum scores for combined segments.

for $n=2 \rightarrow L$ do

for $i=1 \rightarrow L-n+1$ do

$j \leftarrow i+n-1$

for $k=i \rightarrow j-1$ do

// Remove clear-sky power ramps. $S(i, k) \leftarrow(k-i)^{2} \times R(i, k) \times\left[1-R_{C}(i, k)\right]$

$$
\begin{aligned}
& q \leftarrow S(i, k)+J(k, j) \\
& \text { if } q>J[i, j] \text { then } \\
& J[i, j] \leftarrow q \\
& K[k, j] \leftarrow k
\end{aligned}
$$

end for

\section{end for}

\section{end for}

Based on Eqs. (1)-(3), this optimized process can proceed inductively as follows. Assuming that the number of SPREs is $M$ and $\forall m: 1 \leq m<M$, the SPRE interval set $\xi=\left\{E_{m}, \ldots, E_{M}\right\}$ is the set of intervals $E_{m}=\left(s_{m}, e_{m}\right)$ and the non-SPRE interval set $\bar{\xi}$ $=\left\{\overline{E_{m}}, \ldots, \overline{E_{M}}\right\}$ is the set of intervals $\overline{E_{m}}=\left(\overline{s_{m}}, \overline{e_{m}}\right)$. If $\forall\left(\overline{s_{m}}, \overline{e_{m}}\right) \in \bar{\xi}$ and $\forall i, j: \overline{s_{m}}<i<j<\overline{e_{m}}$, then:

$$
\begin{gathered}
R(i, j)=0 \\
S(i, j)=0 \\
J^{*}\left(\overline{s_{m}}, \overline{e_{m}}\right)=0
\end{gathered}
$$

If $\forall\left(s_{m}, e_{m}\right) \in \xi$ and $\forall i, j: s_{m}<i<j<e_{m}$, the objective function $J^{*}\left(s_{m}, e_{m}\right)$ is expressed as:

$$
\begin{aligned}
J^{*}\left(s_{m}, e_{m}\right) & =\max _{s_{m}<k_{1}<e_{m}}\left[S\left(s_{m}, k_{1}\right)+J\left(k_{1}+1, e_{m}\right)\right] \\
& =\max _{s_{m}<k_{1}<e_{m}} S\left(s_{m}, k_{1}\right)+\max _{k_{1}+1<k_{2}<e_{m}} S\left(k_{1}+1, k_{2}\right)+\cdots \\
& +\max _{k_{i-1}+1<k_{i}<e_{m}} S\left(k_{i-1}, k_{i}\right)+J\left(k_{i}+1, e_{m}\right) \\
=\sin _{m}<k_{1}<k_{2} & \max _{-1<k_{i}<e_{m}} S\left(s_{m}, k_{1}\right)+S\left(k_{1}+1, k_{2}\right)+\cdots S\left(k_{i-1}, k_{i}\right)
\end{aligned}
$$

Thus, considering Eq. (2), $J^{*}\left(s_{m}, e_{m}\right)$ is: $J^{*}\left(s_{m}, e_{m}\right)=S\left(s_{m}\right.$, $\left.e_{m}\right)$. Noted that there are only two possible cases (SPRE $\left(E_{1}\right)$ or non-SPRE $\left.\left(\overline{E_{1}}\right)\right)$ for the initial event. Thus, an optimal event sequence of SPREs and non-SPREs can be presented as $\Theta=\left\{E_{m}\right.$, $\left.\overline{E_{m}}, E_{m+1}, \overline{E_{m+1}}, \ldots, E_{M}, \overline{E_{M}}\right\}$ or $\left\{\overline{E_{m}}, E_{m+1}, \overline{E_{m+1}}, \ldots, E_{M}, \overline{E_{M}}\right\}$ for a given $L$ solar power series. If a solar power series $p_{s_{m}}, \ldots$, $p_{\overline{\bar{e}_{M}}}$ with the event sequence $\Theta=\left\{E_{m}, \overline{E_{m}}, E_{m+1}, \overline{E_{m+1}}, \ldots, E_{M}\right.$, $\left.\overline{E_{M}}\right\}$ has a solution to Eq. (1) being $J^{*}\left(s_{m}, \overline{e_{M}}\right)$, the solution is expressed in Eq. (6) and Eq. (8); if a solar power series $p_{\overline{s_{m}}}, \ldots$, $p_{\overline{e_{M}}}$ with the event sequence $\Theta=\left\{\overline{E_{m}}, E_{m+1}, \overline{E_{m+1}}, \ldots, E_{M}, \overline{E_{M}}\right\}$ has a solution to Eq. (1) being $J^{*}\left(\overline{s_{m}}, \overline{e_{M}}\right)$, the solution is expressed in Eq. (7) and Eq. (9):

$$
\begin{aligned}
J^{*}\left(s_{m}, \overline{e_{M}}\right) & =\max _{s_{m}<k_{1} \leq e_{m}}\left[S\left(s_{m}, k_{1}\right)+J\left(k_{1}+1, \overline{e_{M}}\right)\right] \\
& =\max _{s_{m}<k_{1} \leq e_{m}}\left\{S\left(s_{m}, k_{1}\right)+\max _{k_{1}+1<k_{2} \leq e_{m}}\left[S\left(k_{1}+1, k_{2}\right)+J\left(k_{2}+1, \overline{e_{M}}\right)\right]\right\} \\
& =\max _{s_{m}<k_{1} \leq e_{m}}\left\{S\left(s_{m}, k_{1}\right)+\max _{k_{1}+1<k_{2} \leq e_{m}}\left\{S\left(k_{1}+1, k_{2}\right)+\cdots+\max _{k_{i-1}+1<k_{i} \leq e_{m}}\left[S\left(k_{i-1}, k_{i}\right)+J\left(k_{i}+1, \overline{e_{M}}\right)\right]\right\}\right\} \\
& =\max _{s_{m}<k_{1}<k_{2}<\cdots<k_{i-1}<k_{i} \leq e_{m}}\left\{S\left(s_{m}, k_{1}\right)+S\left(k_{1}+1, k_{2}\right)+\cdots+S\left(k_{i-1}, k_{i}\right)\right\}+\max _{k_{i-1}+1<k_{i} \leq e_{m}} J\left(k_{i}+1, \overline{e_{M}}\right) \\
J^{*}\left(\overline{s_{m}}, \overline{e_{M}}\right) & =\max _{\overline{s_{m}}<k_{1} \leq \overline{e_{m}}}\left[S\left(\overline{s_{m}}, k_{1}\right)+J\left(k_{1}+1, \overline{e_{M}}\right)\right] \\
& =\max _{\overline{s_{m}}<k_{1} \leq \overline{e_{m}}}\left\{S\left(\overline{s_{m}}, k_{1}\right)+\max _{k_{1}+1<k_{2} \leq \overline{e_{m}}}\left[S\left(k_{1}+1, k_{2}\right)+J\left(k_{2}+1, \overline{e_{M}}\right)\right]\right\} \\
& =\max _{\overline{s_{m}}<k_{1} \leq \overline{e_{m}}}\left\{S\left(\overline{s_{m}}, k_{1}\right)+\max _{k_{1}+1<k_{2} \leq \overline{e_{m}}}\left\{S\left(k_{1}+1, k_{2}\right)+\cdots+\max _{k_{i-1}+1<k_{i} \leq \overline{e_{m}}}\left[S\left(k_{i-1}, k_{i}\right)+J\left(k_{i}+1, \overline{e_{M}}\right)\right]\right\}\right\} \\
& =\max _{\overline{s_{m}}<k_{1}<k_{2}<\cdots<k_{i-1}<k_{i} \leq \overline{e_{m}}}\left\{S\left(\overline{s_{m}}, k_{1}\right)+S\left(k_{1}+1, k_{2}\right)+\cdots+S\left(k_{i-1}, k_{i}\right)\right\}+\underset{k_{i-1}+1<k_{i} \leq \overline{e_{m}}}{J\left(k_{i}+1, \overline{e_{M}}\right)}
\end{aligned}
$$


Thus, considering Eq. (2), $J^{*}\left(s_{m}, \overline{e_{M}}\right)$ in Eq. (6) can be transformed to:

$$
J^{*}\left(s_{m}, \overline{e_{M}}\right)=S\left(s_{m}, e_{m}\right)+J^{*}\left(\overline{s_{m}}, \overline{e_{M}}\right)
$$

Likewise, considering Eq. (4), $J^{*}\left(\overline{s_{m}}, \overline{e_{M}}\right)$ in Eq. (7) is:

$$
\begin{aligned}
J^{*}\left(\overline{s_{m}}, \overline{e_{M}}\right) & =\max _{\overline{s_{m}<k_{1}<k_{2}<\cdots<k_{i-1}<k_{i} \leq \overline{e_{m}}}} J\left(k_{i}+1, \overline{e_{M}}\right) \\
& =J^{*}\left(s_{m+1}, \overline{e_{M}}\right)
\end{aligned}
$$

If a solar power series is $p_{s_{1}}, \ldots, p_{\overline{e_{M}}}$, the solution is:

$$
J^{*}\left(s_{1}, \overline{e_{M}}\right)=\sum_{m=1}^{M} S\left(s_{m}, e_{m}\right)
$$

If a solar power series is $p_{\overline{s_{1}}}, \ldots, p_{\overline{\bar{Q}_{M}}}$, the solution is:

$$
J^{*}\left(\overline{s_{1}}, \overline{e_{M}}\right)=\sum_{m=2}^{M} S\left(s_{m}, e_{m}\right)
$$

Fig. 3 shows $M$ sliding windows with the start point $\left(t_{w s}, x_{w s}\right)$ and the end point $\left(t_{w e}, x_{w e}\right)$. A set of significant ramps $E_{i}$ will be detected in each window in which $E_{i}=\left(s_{i}, e_{i}\right)$ with the start $\left(s_{i}\right)$ and end $\left(e_{i}\right)$. The number of significant ramps in each window may be different (e.g., the first window with $\mathrm{L}_{1}$ and the last window with $\mathrm{L}_{\mathrm{M}}$ ).

In brief, all the segments (represented by the square points in Fig. 2) are first extracted by the SDA with a predefined parameter $\varepsilon$. Then all extracted segments are input into the optimization procedure (the red block shown in Fig. 3). The extracted segments are merged to yield a set of optimized significant ramp events $\left(s_{1}, e_{1}\right), \ldots,\left(s_{\mathrm{L}}, e_{\mathrm{L}}\right)$. During this procedure, a comparison process (the blue block shown in Fig. 3 ) is deployed to remove the SPREs occurring in both the measured and clear-sky solar power generations. A case study of the OpSDA is shown in the next section.

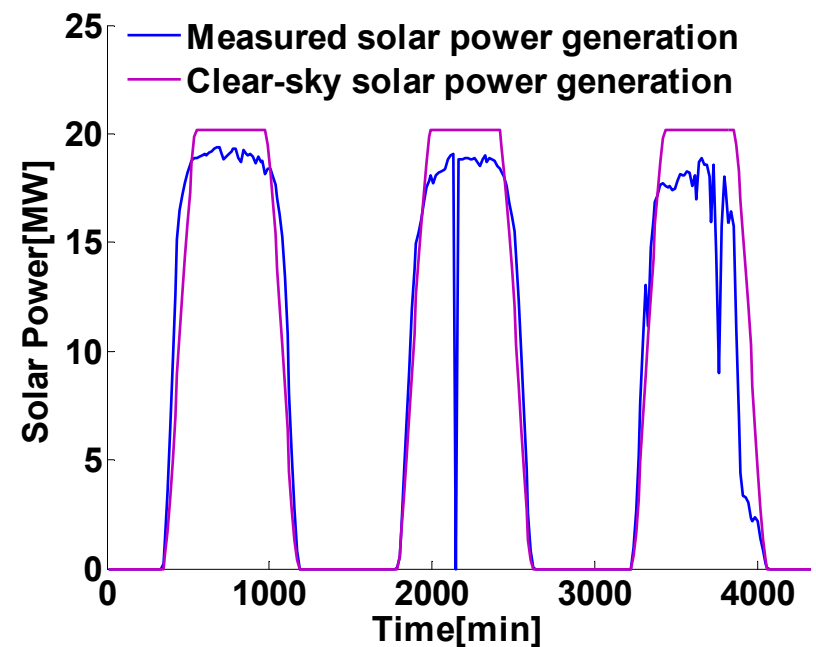

FIGURE 4. SAMPLE INTERVAL OF SOLAR POWER TIME SERIES IN TEP DATA

\section{EXPERIMENTAL RESULTS}

\section{Data Description}

In this section, we present various statistics to analyze the proposed solar ramp detection method. The solar power data collected from TEP is used for solar power ramp detection. The rated capacity of the TEP solar is $25 \mathrm{MW}$. The data includes both the measured power generation and the corresponding clear-sky power generation sampled every 15 minutes. The time interval of the TEP data spans from June 1, 2013, to October 30,2013, including 14,564 samples. An example of the solar data time series is shown in Fig. 4.

\section{OpSDA Ramp Event Detection Results}

OpSDA was compared to a ramp detection method proposed by Sevlian and Rajagopal $[22,28]$, referred to as the L1-SW. The L1-SW method was developed for wind power ramp event detection, which can characterize the ramp start times, durations, and rates as well as other key features needed in the operation of a power system. In this study, the L1-SW was modified to extract SPREs. The segregation process of the L1SW method uses L1 trend fitting with a penalty parameter $\lambda$ and the second derivative with a threshold $\gamma$; whereas the OpSDA uses the SDA for segregation. The L1-SW method is capable of smoothing the noise with preprocessing in solar power and subsequently segregating the solar power into piecewise data. In this subsection, a significant ramp is defined as the change in solar power that is greater than $10 \%$ of the installed solar capacity. Note that this threshold $(10 \%)$ is set relatively smaller to extract sufficient ramps to compare the L1SW to the OpSDA.

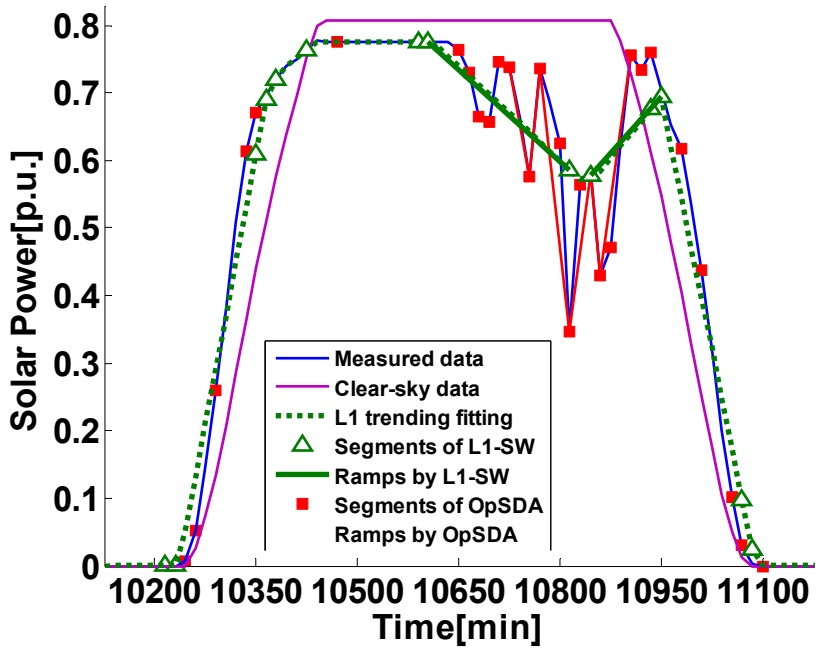

FIGURE 5. COMPARISON RESULTS OF SEGMENTS AND SIGNIFICANT RAMPS OF THE L1-SW WITH $\lambda=0.5, \gamma$ $=1 \times 10^{-4}$ TO THE OPSDA WITH $\varepsilon=9 \times 10^{-3}$

Figs. 5 and 6 compare the segments approximated by the L1-SW and OpSDA methods to different parameter values. The parameter $\varepsilon$ in the OpSDA is set to be $9 \times 10^{-3}$ in both Figs. 5 and 6; the parameter $\lambda$ in L1-SW is set to be 0.5 and 0.02 in Figs. 5 and 6, respectively; the parameter $\gamma$ in L1-SW is set to be $1 \times 10^{-4}$ and $5 \times 10^{-6}$, respectively. Significant ramps extracted by the two methods are also shown in Figs. 5 and 6 . 
Fig. 5 indicates that segments of the OpSDA are more accurate than those of the L1-SW with larger $\lambda$ and $\gamma$. For example, as shown in the intervals of the $10,600^{\text {th }}$ to $10,850^{\text {th }}$ minute and the $10,850^{\text {th }}$ to $10,950^{\text {th }}$ minute, the L1-SW method deviates from the measured solar power signal with larger $\lambda$ and $\gamma$. Thus, the OpSDA has a better ramp detection performance than the L1-SW in Fig. 5. By reducing the values of $\lambda$ and $\gamma$, the L1-SW method in Fig. 6 can match the measured solar power signal more precisely. However, this comes with increased computational burden.

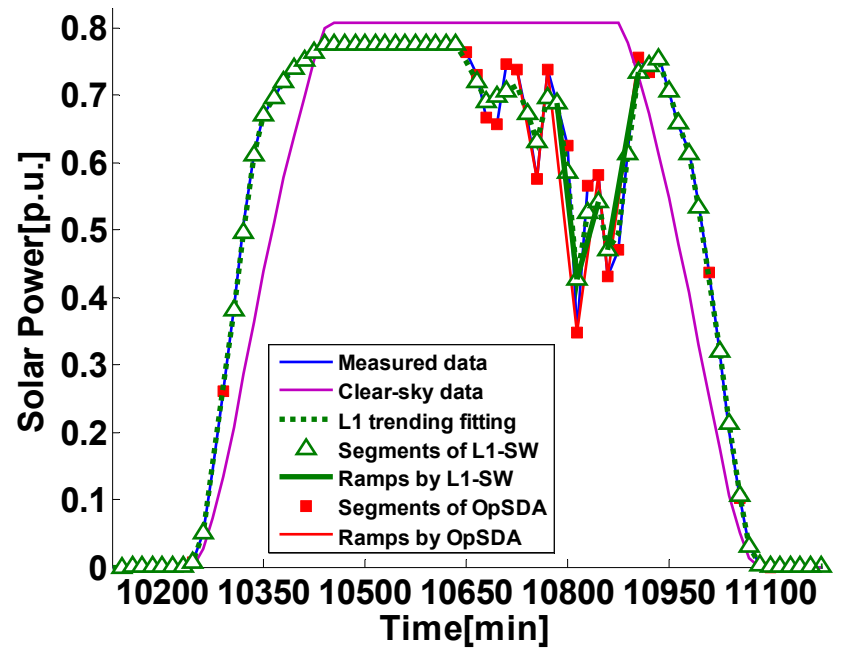

FIGURE 6. COMPARISON RESULTS OF SEGMENTS AND SIGNIFICANT RAMPS OF THE L1-SW WITH $\lambda=0.02, \gamma$ $=5 \times 10^{-6}$ TO THE OPSDA WITH $\varepsilon=9 \times 10^{-3}$

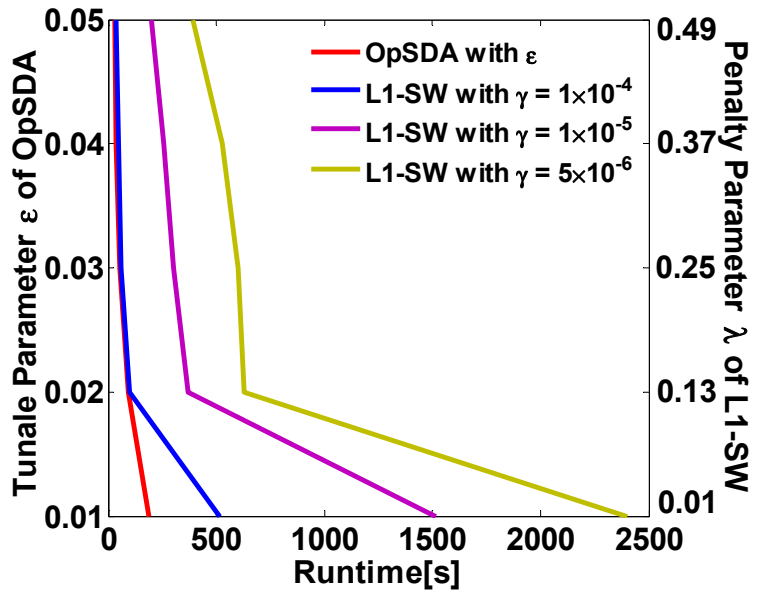

FIGURE 7. RUNTIME COMPARISON OF THE L1-SW WITH THE PARAMETER $\lambda \in[0.01,0.49], \gamma=\left[1 \times 10^{-4}, 1 \times 10^{-5}\right.$, $\left.5 \times 10^{-6}\right]$ TO THE OPSDA WITH THE TUNABLE PARAMETER $\varepsilon \in[0.01,0.05]$

Fig. 7 compares the runtime of the two methods: the OpSDA on the left $\mathrm{y}$-axis and the L1-SW on the right $\mathrm{y}$-axis. Although the accuracy of the L1-SW was improved in Fig. 6 with smaller $\lambda$ and $\gamma$, the runtime of the L1-SW is approximatively 40 minutes. The runtime of the OpSDA with $\varepsilon=9 \times 10^{-3}$ is only 2.5 minutes. Overall, both the ramp detection performance and the runtime of the L1-SW are sensitive to the penalty parameter $\lambda$ and the threshold $\gamma$. The detection performance and runtime of the OpSDA are less sensitive to the parameter. Table 1 lists the number of significant up- and down-ramps in the TEP solar power data according to the three ramp extraction methods and three definitions.

\section{TABLE 1. NUMBER OF OBSERVED RAMPS}

\begin{tabular}{|c|c|c|c|}
\hline & Methods & Up-Ramps & Down-Ramps \\
\hline \multirow{3}{*}{ Definition 1 } & SDA & 169 & 174 \\
\cline { 2 - 4 } & L1-SW & 328 & 327 \\
\cline { 2 - 4 } & OpSDA & 270 & 296 \\
\hline \multirow{3}{*}{ Definition 2 } & SDA & 195 & 201 \\
\cline { 2 - 4 } & L1-SW & 327 & 326 \\
\cline { 2 - 4 } & OpSDA & 314 & 296 \\
\hline \multirow{3}{*}{ Definition 3 } & SDA & 195 & 222 \\
\cline { 2 - 4 } & L1-SW & 327 & 388 \\
\cline { 2 - 4 } & OpSDA & 314 & 322 \\
\hline
\end{tabular}

Table 1 shows that in all three definitions, the SDA method detects the least quantity of SPREs. Basically, there are no combinations of adjacent segments in the SDA, which makes only separate ramps conforming to specific definitions extracted. As for the L1-SW, the most quantity of SPREs is detected with smaller parameters. Consequently, this process needs the most computation. Therefore, the OpSDA detects more quantity than the SDA but with less computation than the L1-SW.

\section{Distribution of Ramp Features}

A numerical statistical distribution of the three key ramp features (ramp duration, rate, and magnitude) based on the TEP solar power data is shown in Figs. 8-10. As for ramp durations, it is observed that the most possible ramp durations detected by all three methods (SDA, OpSDA, and L1-SW) are distributed around 30 minutes with Definition 1; whereas the ramp durations are mostly distributed from 30 minutes to 60 minutes in both Definition 2 and Definition 3. Meanwhile, as for ramp rates, it is observed that the most possible ramp rates are distributed between 6 p.u./min and 13 p.u./min using all three significant ramp definitions. Moreover, this is because solar power fluctuates sharply within a short time (30 minutes or 60 minutes), as shown in Figs. 8-10.

However, as for ramp magnitudes, even though most ramp magnitudes by the three methods are distributed around 0.2 p.u., the empirical distributions derived from the L1-SW and the OpSDA are very close to each other and far from the distributions by the SDA. A sharp peak occurs when using the SDA, which indicates that there are many small solar power ramps. However, the magnitude distributions with the L1-SW and OpSDA present fat tails, which means that the magnitudes of the ramps are increased and optimized by the dynamic programming approach. 


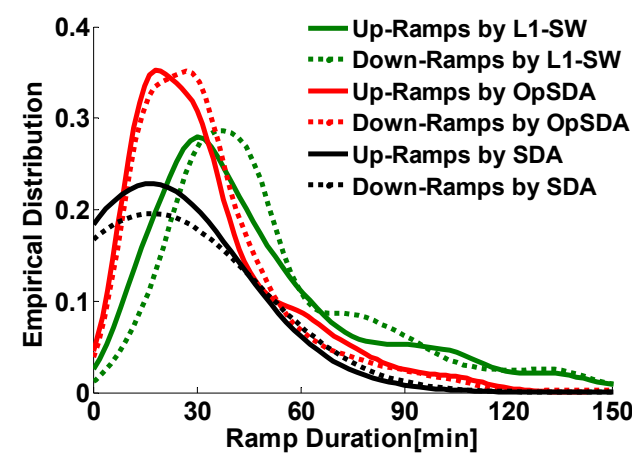

(a) Ramp duration

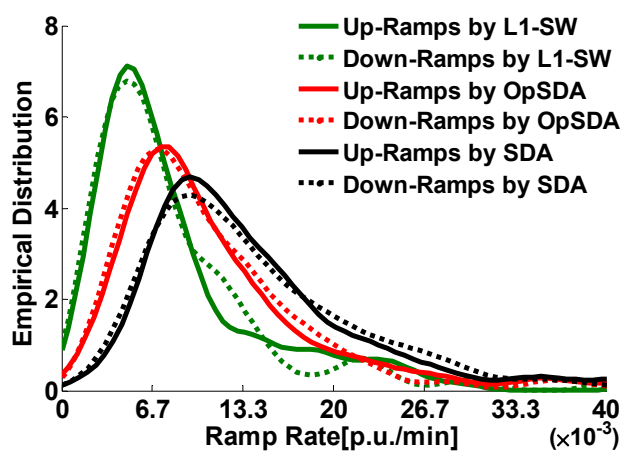

(b) Ramp rate

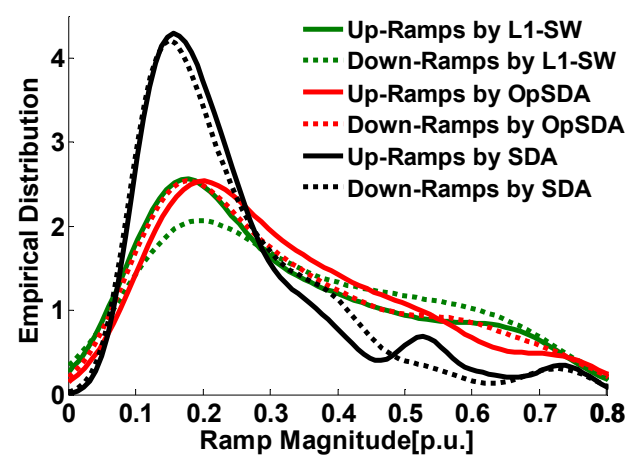

(c) Ramp magnitude

FIGURE 8. RAMP DEFINITION 1: RAMP MAGNITUDE ONLY

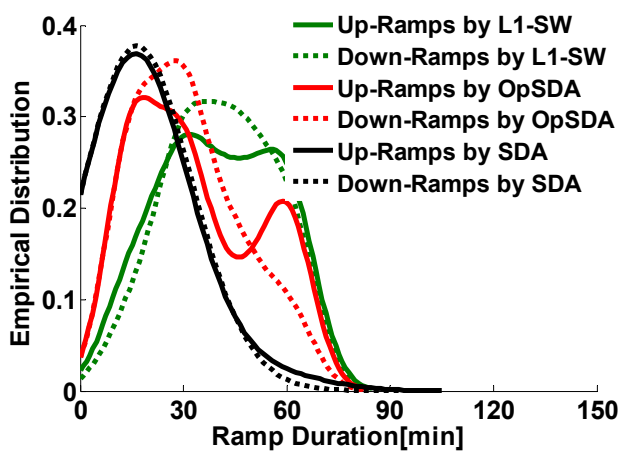

(a) Ramp duration

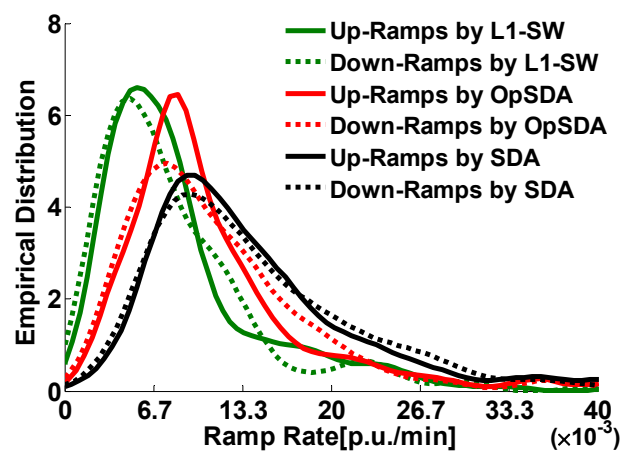

(b) Ramp rate

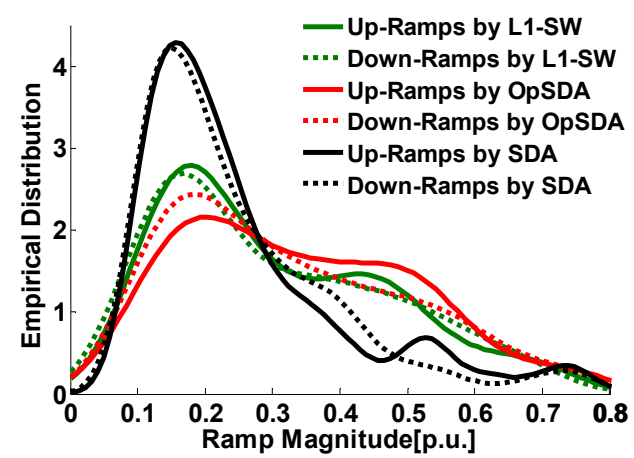

(c) Ramp magnitude

FIGURE 9. RAMP DEFINITION 2: RAMP MAGNITUDE AND RAMP DURATION

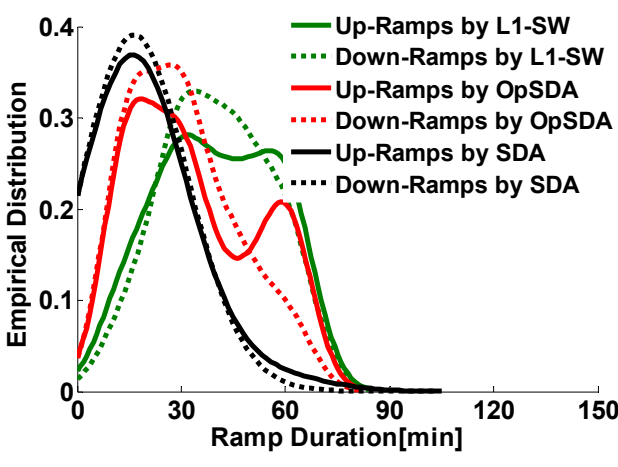

(a) Ramp duration

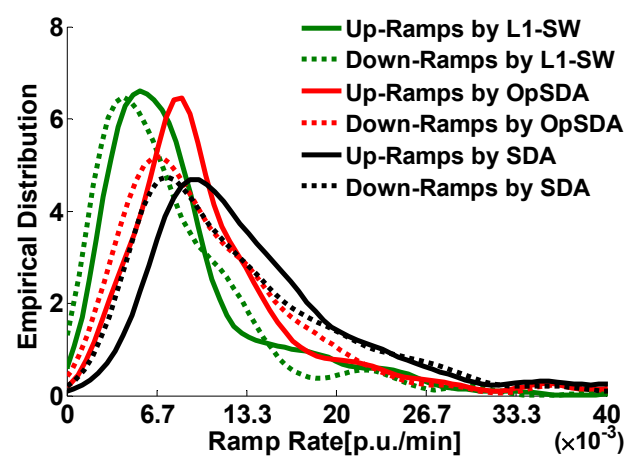

(b) Ramp rate

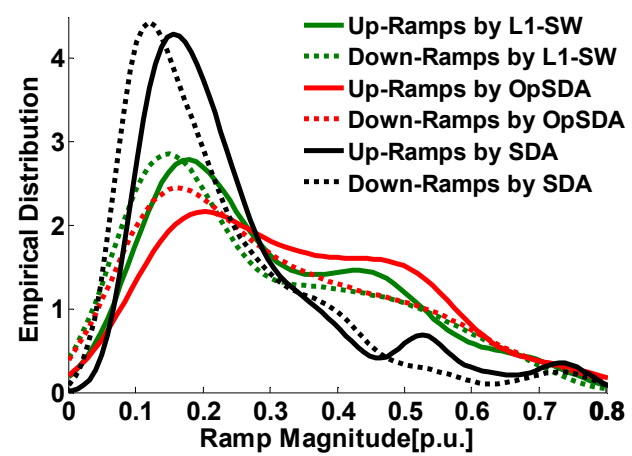

(c) Ramp magnitude

FIGURE 10. RAMP DEFINITION 3: RAMP DIRECTION, RAMP MAGNITUDE, AND RAMP DURATION

\section{APPLICATION OF THE OPSDA FOR TUNING THE PARAMETER $\varepsilon$}

The SDA has the advantage of computational and structural simplicity, which is favorable considering its robustness even with noisy data [17]. Sometimes in online applications, an SDA without any optimization is more preferable because of its inexpensive computation. Currently, this tunable parameter $\varepsilon$ is normally determined through computational experiments. It would be uniquely helpful to develop an optimal strategy for adaptively selecting the best $\varepsilon$ values at different time periods. Toward this end, we propose using the OpSDA as a baseline to determine the optimal parameter value, $\varepsilon_{\text {opt }}$. The solving procedure is to find the $\varepsilon_{\text {opt }}$ that enables the ramps detected by the SDA close to that detected by the OpSDA.
Generally, a smaller $\varepsilon$ value corresponds to smaller fitting errors between the SDA approximation and the measured solar power. However, an $\varepsilon$ value that is too small may segregate a single significant ramp into multiple small ramps that do not satisfy the SPRE ramp definitions.

To determine the optimal parameter, $\varepsilon_{\mathrm{opt}}$, based on the OpSDA, a suite of SPRE detection metrics were used to evaluate the performance of ramp extraction with different $\varepsilon$ values. The adopted metrics include the probability of detection (POD), the critical success index (CSI), the frequency bias score (FBIAS), and the success ratio. These metrics are calculated based on a contingency table in Table 2 that provides a measure of skill for the SDA to approach the OpSDA. True positive (TP) represents the number of ramps detected by the 
SDA that are accurately detected by the OpSDA; false positive (FP) is the number of ramps detected by the SDA that are not detected by the OpSDA; false negative (FN) represents the number of ramps detected by the OpSDA that are not extracted by the SDA; true negative (TN) is the number of nonoccurring events for both the SDA and the OpSDA; and $\mathrm{N}$ is the total number of SPREs.

TABLE 2. CONTINGENCY TABLE FOR THE SDA AND OPSDA

\begin{tabular}{|c|c|c|c|}
\hline & $\begin{array}{c}\text { OpSDA } \\
\text { YES }\end{array}$ & $\begin{array}{c}\text { OpSDA } \\
\text { NO }\end{array}$ & Total \\
\hline SDA YES & TP(hits $)$ & $\mathrm{FP}($ false alarm $)$ & $\mathrm{TP}+\mathrm{FP}$ \\
\hline SDA NO & $\mathrm{FN}($ misses $)$ & $\mathrm{TN}$ & $\mathrm{FN}+\mathrm{TN}$ \\
\hline Total & $\mathrm{TP}+\mathrm{FN}$ & $\mathrm{FP}+\mathrm{TN}$ & $\mathrm{N}=\mathrm{TP}+\mathrm{FP}+\mathrm{FN}+\mathrm{TN}$ \\
\hline
\end{tabular}

Categorical statistics provide measures of accuracy and skill for forecasting in many applications, such as ramps in power, temperature, or rainfall. Based on the contingency table, a suite of metrics can be derived for optimal $\varepsilon$ performance

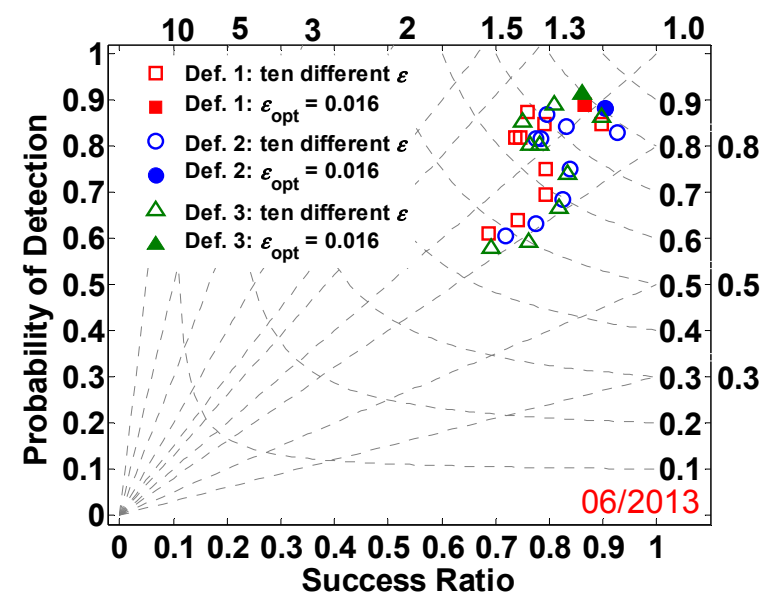

(a)

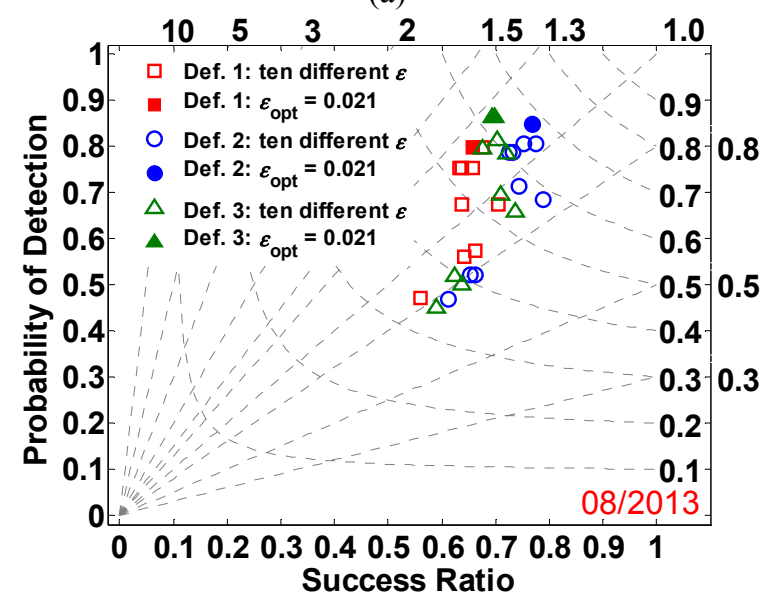

(c) evaluation. The equations of POD, CSI, FBIAS, and FAR can be found in [16] and [29].

After calculating all of the metrics (POD, CSI, FBIAS, and FAR), the optimal $\varepsilon$ value is determined by the largest POD, which can be visualized on the performance diagram shown in Fig. 11. A performance diagram is used to understand the evolution of the SDA with different $\varepsilon$ values. For the performance diagram shown in Fig. 11, (i) the left axis represents the value of POD; (ii) the bottom axis represents the success ratio; (iii) the diagonal dashed lines represent FBIAS; and (iv) the dashed curves show CSI. Fig. 11 shows a performance space of $10 \varepsilon$ values by utilizing each ramp definition. These 10 parameters are derived from 0.006 to 0.096 uniformly. To find the optimal parameter, $\varepsilon_{\mathrm{opt}}$, the points in Fig. 11 move toward the top right corner of the performance diagram. Fig. 11 shows the optimal parameters for four months (June, July, August, and September 2013) using 15-minute resolution data based on three significant solar power ramp definitions.

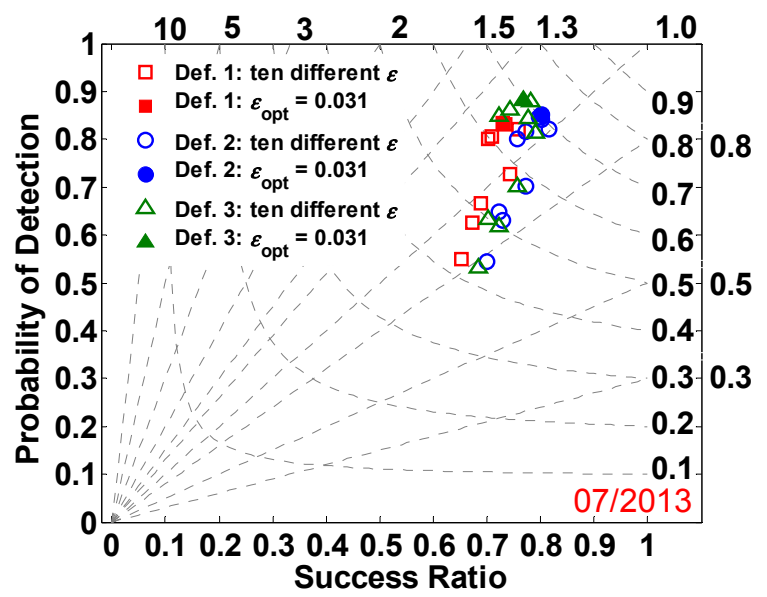

(b)

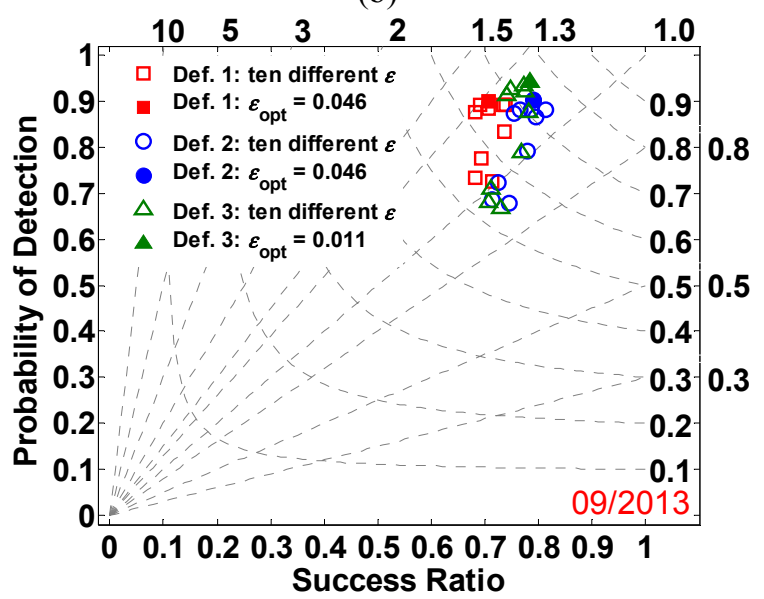

(d)

FIGURE 11. RAMP DETECTION PERFORMANCE WITH THE OPTIMAL PARAMETERS. THE SUBFIGURES (A), (B), (C), AND (D) SHOW OPTIMAL PARAMETERS DEFINED BY THREE RAMP DEFINITIONS IN JUNE, JULY, AUGUST, AND SEPTEMBER 2013, RESPECTIVELY 
It is observed in Fig. 11 that in June, July, and August, the optimal parameters have the same values under the three definitions: $0.016,0.031$, and 0.021 , respectively. This means that the optimal $\varepsilon_{\text {opt }}$ is not sensitive to the ramp definitions in these three months; the determined optimal values are robust. Meanwhile, when using the optimal parameter, $\varepsilon_{\text {opt }}$, both the POD and SR values are larger than 0.7 in all three months; the FBAIS values are close to 1 ; and the CSI values are larger than 0.6 . This shows that the ramp detection results obtained by the SDA with $\varepsilon_{\text {opt }}$ can successfully approach the results obtained by the OpSDA. In September, Definition 3 has a smaller $\varepsilon_{\text {opt }}$ 0.011 than Definition 1 and Definition 2, in which $\varepsilon_{\mathrm{opt}}=0.046$. This can guide power system operators to make decisions about how to best capture solar power ramps.

\section{CONCLUSION}

An OpSDA was developed in this paper for solar power ramp detection. The developed OpSDA was compared to the SDA and L1-SW methods in a case study of solar power ramp extraction from TEP data. The results showed that the OpSDA successfully identified SPREs and performed significantly better than the SDA. The OpSDA provided equal-to-better performance than the L1-SW method with much less computational time. The developed OpSDA was also used as a baseline to determine the optimal values of the tunable parameter in the SDA.

In future work, more data at multiple spatial and temporal resolutions will be analyzed using this ramp detection method OpSDA for further performance evaluation. In addition, some sufficiently small non-SPREs (termed "bumps")—which have very short time durations, small ramp magnitudes, and opposite ramping directions - can abruptly interrupt the iteration of dynamic programming. Future work will merge bumps into the adjacent segments in the process of dynamic programming for better ramp detection.

\section{ACKNOWLEDGMENTS}

This work was supported by the U.S. Department of Energy under Contract No. DE-AC36-08-GO28308 with the National Renewable Energy Laboratory and by the National Basic Research Program of China (2012CB215101).

\section{REFERENCES}

[1] Yan, R., and Saha, T. K., 2012, "Voltage Variation Sensitivity Analysis for Unbalanced Distribution Networks due to Photovoltaic Power Fluctuations," IEEE Transactions on Power Systems, 27(2), pp. 1078-1089.

[2] Lave, M., and Kleiss1, J., 2010, "Solar Variability of Four Sites Across the State of Colorado," Renewable Energy, 35(12), pp. 2867-2873.

[3] Yan, R., and Saha, T. K., 2011, "Investigation of Voltage Variations in Unbalanced Distribution Systems due to High Photovoltaic Penetrations," IEEE Power \& Energy Society General Meeting, San Diego, CA.

[4] Godfrey, T., Mullen, S., Dugan, R. C., Rodine, C., Griffith, D. W., and Golmie, N., 2010, "Modeling Smart Grid Applications with Co-Simulation," 1st IEEE International Conference on Smart Grid Communications, Gaithersburg, MD.
[5] Cui, M., Ke, D., Sun, Y., Gan, D., Zhang, J., and Hodge, B.M., 2015, "Wind Power Ramp Event Forecasting Using a Stochastic Scenario Generation Method," IEEE Transactions on Sustainable Energy, DOI: 10.1109/TSTE.2014.2386870.

[6] Zhang, J., Florita, A., Hodge, B.-M., Lu, S., Hamann, H. F., Banunarayanan, V., and Brockway, A., 2015, "A Suite of Metrics for Assessing the Performance of Solar Power Forecasting," Solar Energy, 111, pp. 157-175.

[7] Bacher, P., Madsen, H., and Nielsen, H. A., 2009, "Online Short-Term Solar Power Forecasting," Solar Energy, 83(10), pp. 1772-1783.

[8] Bessa, R. J., Trindade A., and Miranda, V., 2015, "SpatialTemporal Solar Power Forecasting for Smart Grids," IEEE Transactions on Industrial Informatics, 11(1), pp. 232-241.

[9] Yang, H. T., Huang, C. M., Huang, Y. C., and Pai, Y. S., 2014, "A Weather-Based Hybrid Method for 1-Day Ahead Hourly Forecasting of PV Power Output," IEEE Transactions on Sustainable Energy, 5(3), pp. 917-926.

[10] Yang, C., Thatte, A. A., and Xie, L., 2015, "Multitime-Scale Data-Driven Spatio-Temporal Forecast of Photovoltaic Generation," IEEE Transaction on Sustainable Energy, 6(1), pp. 104-112.

[11] Florita, A., Hodge, B.-M., and Orwig, K., 2013, "Identifying Wind and Solar Ramping Events," IEEE 5th Green Technologies Conference, Denver, CO.

[12] Hummon, M., Cochran, J., Weekley, A., Lopez, A., Zhang, J., Stoltenberg, B., Parsons, B., Batra, P., Mehta, B., and Patel, D., 2014, "Variability of Photovoltaic Power in the State of Gujarat Using High Resolution Solar Data," Technical Report, No. NREL/TP-7A40-60991, National Renewable Energy Laboratory, Golden, CO.

[13] Hodge, B.-M., Hummon, M., and Orwig, K., 2011, "Solar Ramping Distributions over Multiple Timescales and Weather Patterns," 1st International Workshop on Integration of Solar Power into Power Systems, Aarhus, Denmark.

[14] Sengupta, M., and Keller, J., 2012, "PV Ramping in a Distributed Generation Environment: A Study Using Solar Measurements," 38th IEEE Photovoltaic Specialists Conference, Austin, TX.

[15] Bristol, E. H., 1990, "Swinging Door Trending: Adaptive Trend Recording?" ISA National Conference Proceedings.

[16] Zhang, J., Florita, A., Hodge, B.-M., and Freedman, J., 2014, "Ramp Forecasting Performance From Improved Short-Term Wind Power Forecasting," ASME International Design Engineering Technical Conference \& Computers and Information in Engineering Conference, Buffalo, NY.

[17] Makarov, Y. V., Loutan, C., Ma, J., and Mello, P., 2009, "Operational Impacts of Wind Generation on California Power Systems," IEEE Transactions on Power System, 24(2), pp. 1039-1050.

[18] Ma, J., Makarov, Y. V., Loutan, C., and Xie, Z., 2011, "Impact of Wind and Solar Generation on the California ISO's IntraHour Balancing Needs," IEEE Power \& Energy Society General Meeting, San Diego, CA.

[19] Ma, J., Lu, S., Etingov, P. V., and Makarov, Y. V., 2012, "Evaluating the Impact of Solar Generation on Balancing Requirements in Southern Nevada System," IEEE Power \& Energy Society General Meeting, San Diego, CA.

[20] Ma, J., Lu, S., Hafen, R. P., Etingov, P. V., Makarov, Y. V., and Chadliev, V., 2012, "The Impact of Solar Photovoltaic Generation on Balancing Requirements in the Southern Nevada System," IEEE Transmission and Distribution Conference and Exposition, Orlando, FL. 
[21] Cui, M., Zhang, J., Florita, A. R., Hodge, B.-M., Ke, D., and Sun, Y., 2015, "An Optimized Swinging Door Algorithm for Wind Power Ramp Events Detection," IEEE Power \& Energy Society General Meeting, Denver, CO.

[22] Sevlian, R., and Rajagopal, R., 2013, "Detection and Statistics of Wind Power Ramps," IEEE Transactions on Power System, 28(4), pp. 3610-3620.

[23] Boulaxis, N. G., and Papadopoulos, M. P., 2002, "Optimal Feeder Routing in Distribution System Planning Using Dynamic Programming Technique and GIS Facilities," IEEE Transactions on Power Delivery, 17(1), pp. 242-247.

[24] Marano, V., Rizzo, G., and Tiano, F. A., 2012, "Application of Dynamic Programming to the Optimal Management of a Hybrid Power Plant with Wind Turbines, Photovoltaic Panels and Compressed Air Energy Storage," Applied Energy, pp. 849-859.
[25] Barr, D. C., 1994, "The Use of a Data Historian to Extend Plant Life," International Conference on Life Management of Power Plants, Edinburgh, U.K.

[26] Kamath, C., 2010, "Understanding Wind Ramp Events Through Analysis of Historical Data," IEEE Power \& Energy Society Transmission and Distribution Conference and Exposition, New Orleans, LA.

[27] Potter, C. W., Grimit, E., and Nijssen, B., 2009, "Potential Benefits of a Dedicated Probabilistic Rapid Ramp Event Forecast Tool," IEEE Power \& Energy Society Power Systems Conference and Exposition, Seattle, WA.

[28] Sevlian, R., and Rajagopal, R., 2012, "Wind Power Ramps: Detection and Statistics," IEEE Power \& Energy Society General Meeting, San Diego, CA.

[29] Roebber, P. J., 2009, "Visualizing Multiple Measures of Forecast Quality," Weather and Forecasting, 24(2), pp. 601608. 